Oriolus Revista Científica, ISSN: 2215-6100.1 (1). Julio, 2020, pp 1-13

Arias Araya

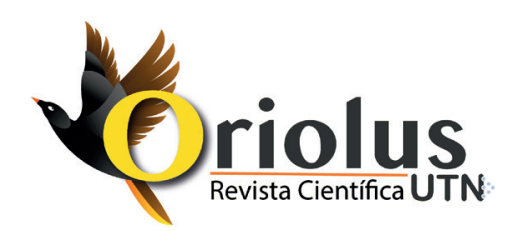

Oriolus Revista Científica

ISSN: 2215-6100

revista.cientifica.oriolus@utn.ac.cr

Universidad Técnica Nacional

\title{
Bioindicadores de contaminación en aguas residuales de sistemas agropecuarios en el Distrito de Riego Arenal Tempisque, Guanacaste, Costa Rica
}

\author{
Bioindicators of pollution in wastewater farming systems in \\ the Arenal Tempisque Irrigation District, Guanacaste, Costa Rica
}

\begin{abstract}
María Fernanda Arias Araya
Bioindicadores de contaminación en aguas residuales de sistemas agropecuarios en el Distrito de Riego Arenal Tempisque, Guanacaste, Costa Rica

Oriolus Revista Científica, Vol. 1, N. ${ }^{\circ} 1,2020$

Universidad Técnica Nacional

Disponible en: http://revistas.utn.ac.cr/index.php/oriolus

Bibliografía:

Arias Araya, María. "Bioindicadores de contaminación en aguas residuales de sistemas agropecuarios en el Distrito de Riego Arenal Tempisque, Guanacaste, Costa Rica". Revista Científica Oriolus 1, N. ${ }^{\circ} 1$ (2020): 1-13.
\end{abstract}


Oriolus Revista Científica, ISSN: 2215-6100.1 (1). Julio, 2020, pp 1-13

Arias Araya

\title{
Bioindicadores de contaminación en aguas residuales de sistemas agropecuarios en el Distrito de Riego Arenal Tempisque, Guanacaste, Costa Rica
}

\section{Bioindicators of pollution in wastewater farming systems in the Arenal Tempisque Irrigation District, Guanacaste, Costa Rica}

María Fernanda Arias Araya

Laboratorio para Aguas y Reproducción de Especies

Dulceacuícolas. Universidad Técnica Nacional, Costa Rica

mfarias@utn.ac.cr

(iD) https://orcid.org/0000-0003-3315-819X
Recepción: 29 de abril del 2019

Aprobación: 11 de febrero del 2020

\section{Resumen}

Los canales de drenaje son una característica prominente de muchas áreas agrícolas de manejo intensivo. Estos pequeños cuerpos de agua poco profundos albergan comunidades de macroinvertebrados, que se asemeja a pequeños ecosistemas lénticos naturales. En este estudio se realizó una evaluación de la comunidad de macroinvertebrados en cuatro drenajes principales (Falconiana, Tamarindo, Bagatzí y La Guaria) y dos secundarios (Lomas y Paso Hondo) del Distrito de Riego Arenal-Tempisque (DRAT) ubicado en la provincia de Guanacaste. Los macroinvertebrados fueron recolectados utilizando el método de sustratos artificiales, los cuales fueron colonizados por los organismos durante los meses de marzo, abril, junio, julio, agosto y noviembre de 2018. Los canales con mayor abundancia de macroinvertebrados fueron Tamarindo, Bagatzí y Falconiana, mayor riqueza Falconiana y La Guaria. Según el Índice BMWP-CR los canales de drenaje presentan aguas de calidad muy mala, extremadamente contaminadas, aguas de calidad mala, muy contaminadas; $y$ aguas de calidad mala, contaminadas.

Palabras claves: Producción, sistemas agroforestales, Bactris guineensis, frutos.

\begin{abstract}
Drainage channels are a prominent feature of many intensively managed agricultural areas. These small shallow bodies of water refuge communities of macroinvertebrates, which resemble small natural lentic ecosystems. In this study, an evaluation of the macroinvertebrate community was carried out in four main drains (Falconiana, Tamarindo, Bagatzí and La Guaria) and two secondary ones (Lomas and Paso Hondo) of the Arenal-Tempisque Irrigation District (DRAT) located in the province of Guanacaste. The macroinvertebrates were collected using the artificial substrates method, which were colonized by the organisms during the months of March, April, June, July, August, and November 2018. The channels with the greatest abundance of macroinvertebrates were Tamarindo, Bagatzí and Falconiana, greater wealth Falconiana and La Guaria. According to the BMWP-CR Index, drainage channels have very poor-quality water, extremely polluted, poor quality water, very polluted; and bad quality water, polluted.
\end{abstract}

Keywords: Bioindicators, Pollution, Wastewater, Agricultural Systems.

Esta obra esta bajo una Licencia de Creative Commons Atribución NoComercial-SinDerivar Internacional 
Oriolus Revista Científica, ISSN: 2215-6100.1 (1). Julio, 2020, pp 1-13

Arias Araya

\section{Introducción}

Los ecosistemas de agua dulce son uno de los más amenazados del mundo, estos ecosistemas han sido afectados por diversas actividades antropogénicas que pueden estar asociadas con la deforestación y las prácticas agropecuarias que alteran de manera indirecta la estructura y función de los macroinvertebrados bentónicos (Vörösmarty et al., 2010).

La agricultura moderna intensiva está acompañada por cambios sustanciales en la estructura del paisaje que a menudo resulta en una disminución del hábitat y la biodiversidad. Sin embargo, los canales de drenaje agrícola en paisajes mal estructurados pueden ofrecer hábitats valiosos para una notable diversidad de organismos asociados al agua (Clarke, 2015).

Los canales son redes que drenan el exceso de agua de lluvia o las filtraciones de aguas subterráneas de los campos agrícolas los cuales se conectan a los ríos y lagos, y viceversa, así mismo, suministran agua a las tierras de cultivo durante los períodos secos (Verdonschot, Keizer-Vlek, Verdonschot, 2012).

Estos cuerpos de agua artificiales han recibido poca atención en estudios ecológicos en el pasado. En los últimos años, cada vez más estudios se centran en el valor potencial de biodiversidad de los canales de drenaje (Armitage, Szoszkiewicz, Blackburn, Nesbitt, 2003; Herzon y Helenius, 2008). Painter (1999) expresa la importancia de los drenajes como punto caliente para los macroinvertebrados acuáticos. Estas aguas son poco conocidas en comparación con la de los fragmentos naturales. Si bien, los ríos son naturales, los canales de drenaje, generalmente, se consideran infraestructura hidrológica en apoyo de la agricultura.

Los macroinvertebrados son considerados como buenos indicadores biológicos, que pueden proporcionar una idea de las condiciones actuales y pasadas de un cuerpo de agua e integrar los efectos de múltiples factores estresantes. En el caso de los canales de drenaje, no existe un estado natural, por lo tanto, el sistema al que se puede hacer referencia podría ser i) la condición más conocida o restante del sistema, una condición histórica o una condición menos perturbada, ii.) la condición potencialmente óptima del sistema, derivada del rango de variables ambientales y otras características del hábitat presentes en un sitio (Verdonschot et al., 2012; Bonada, Prat, Resh y Statzner, 2006).

La riqueza de especies de grupos taxonómicos se considera a menudo como un indicador de calidad ecológica, pero, especialmente, en sistemas con ambientes "duros", las aguas de buena calidad ecológica pueden ser relativamente pobres en el número total de especies, lo que indica la importancia de la identidad de especies junto a la riqueza (Verdonschot et al., 2012; Hanson, Springer y Ramírez, 2010; Vega-Prado, 2004).

La pérdida y fragmentación del hábitat dulceacuícola debido a las actividades agropecuarias y piscícolas es recientemente una de las mayores preocupaciones en torno al recurso hídrico. Por lo que es esencial un cambio en la política ambiental, donde el monitoreo y los estudios no sean exclusivamente para evitar la degradación de la calidad del agua, sino que, además, se haga hincapié en el mantenimiento y mejora del estado global de los ecosistemas acuáticos.

La valoración del estado ecológico de los cursos de aguas superficiales conlleva la evaluación de la integridad de todos los componentes propios del ecosistema fluvial: comunidades biológicas,

Esta obra esta bajo una Licencia de Creative Commons Atribución NoComercial-SinDerivar Internacional 
Oriolus Revista Científica, ISSN: 2215-6100.1(1). Julio, 2020, pp 1-13

Arias Araya

características fisicoquímicas y elementos hidromorfológicos del sistema. Las comunidades de macroinvertebrados han sido seleccionados como uno de los grupos de organismos más relevantes en la valoración del estado ecológico de las masas de agua, ya que han demostrado ser buenos indicadores (Bonada et al., 2006).

En Costa Rica, el uso de macroinvertebrados como bioindicadores en el estudio de la calidad del agua se ha incrementado en la última década y se han aplicado métodos estandarizados en el ámbito nacional (Gutiérrez-Fonseca y Lorion, 2014). De tal manera que es uno de los países tropicales que ha adoptado el monitoreo biológico y adaptado al BMWP para sus cuerpos de agua, el cual se denomina BMWP-CR. Gutiérrez-Fonseca y Lorion (2014) demuestran que el Índice BMWP-CR es sensible y su respuesta dependerá del equipo que se utilice y la intensidad del muestreo (Reglamento N. ${ }^{\circ}$ 33903-MINAE-S (Presidente de la República, Ministerio de Ambiente y Ministra de Salud, 2007).

En el Distrito de Riego Arenal Tempisque confluyen una serie de factores de origen antropogénico que afectan el equilibrio natural de los ecosistemas terrestres y acuáticos. El cambio climático puede tener efectos potenciales significativos en la hidrología del caudal hídrico disponible, se pronostica que la intensificación de los ciclos hidrológicos influya mucho en los procesos limnológicos acentuando la escorrentía. Esto hace que los ecosistemas acuáticos sean más vulnerables a la contaminación, por lo que reciben la mayor cantidad de contaminantes, esto puede provocar un declive de su diversidad biológica. Con este estudio se pretendió determinar qué comunidades de invertebrados son capaces de colonizar sustratos artificiales en los canales y su relación con parámetros físicos y químicos que maneja el drenaje agrícola.

\section{Metodología}

Descripción del sitio. Las aguas del río Arenal se embalsan en Tilarán y luego, a través de la conducción, por el complejo hidroeléctrico ARDESA, pasan de la vertiente Atlántica a la del Pacífico, generan electricidad y abastecen las áreas de riego del DRAT, en el Pacífico Norte. La presa derivadora Miguel Pablo Dengo toma las aguas turbinadas del complejo ARDESA, junto con las del río Magdalena y las distribuye en los canales del sur y del oeste, y deja pasar los excedentes en las épocas de máxima generación hidroeléctrica, dado que no tiene capacidad de almacenamiento. El DRAT se compone de ocho subdistritos: Abangares, Lajas, Cañas, Piedras, Cabuyo, Tempisque, Zapandí Norte y Zapandí Sur. Los mayores meses de demanda de agua en el DRAT son febrero con 49,64 m3 /s, marzo con $55,47 \mathrm{~m} 3 / \mathrm{s}$ y abril con $47,96 \mathrm{~m} 3 / \mathrm{s}$ para riego de cultivos, mientras que las demandas piscícolas son de 19,00 m3/s durante todo el año. Por el canal del Oeste la mayor demanda se presenta en marzo con 38,47 m3/s y mientras que por el canal del Sur es de 17,00 m3/s, durante todo el año (SENARA, 2018). Dentro de los subdistritos se encuentran canales de drenaje, cuya función principal es desaguar los excesos de agua, ya sea por riego o precipitación. Además, son relativamente pequeños (de 2 a $8 \mathrm{~m}$ de ancho, un metro de profundidad) y poseen una estricta linealidad. La hidrología de estos canales es completamente artificial, está ligada a la actividad, tipo de cultivo aledaño y época del año.

El estudio fue realizado en cuatro drenajes principales (Falconiana, Tamarindo, Bagatzí y La Guaria) y dos secundarios (Lomas y Paso Hondo) del Distrito de Riego Arenal-Tempisque (DRAT) ubicado en la provincia de Guanacaste como se muestra en la figura 1, la cual fue realizada utilizando el software libre QGis 2.18.16 y para la georreferenciación de los drenajes se utilizó el GPS garmin Oregon 550. En total fueron muestreados cinco drenajes durante cada mes entre marzo y noviembre de 2018.

Esta obra esta bajo una Licencia de Creative Commons Atribución NoComercial-SinDerivar Internacional 
Oriolus Revista Científica, ISSN: 2215-6100.1 (1). Julio, 2020, pp 1-13

Arias Araya

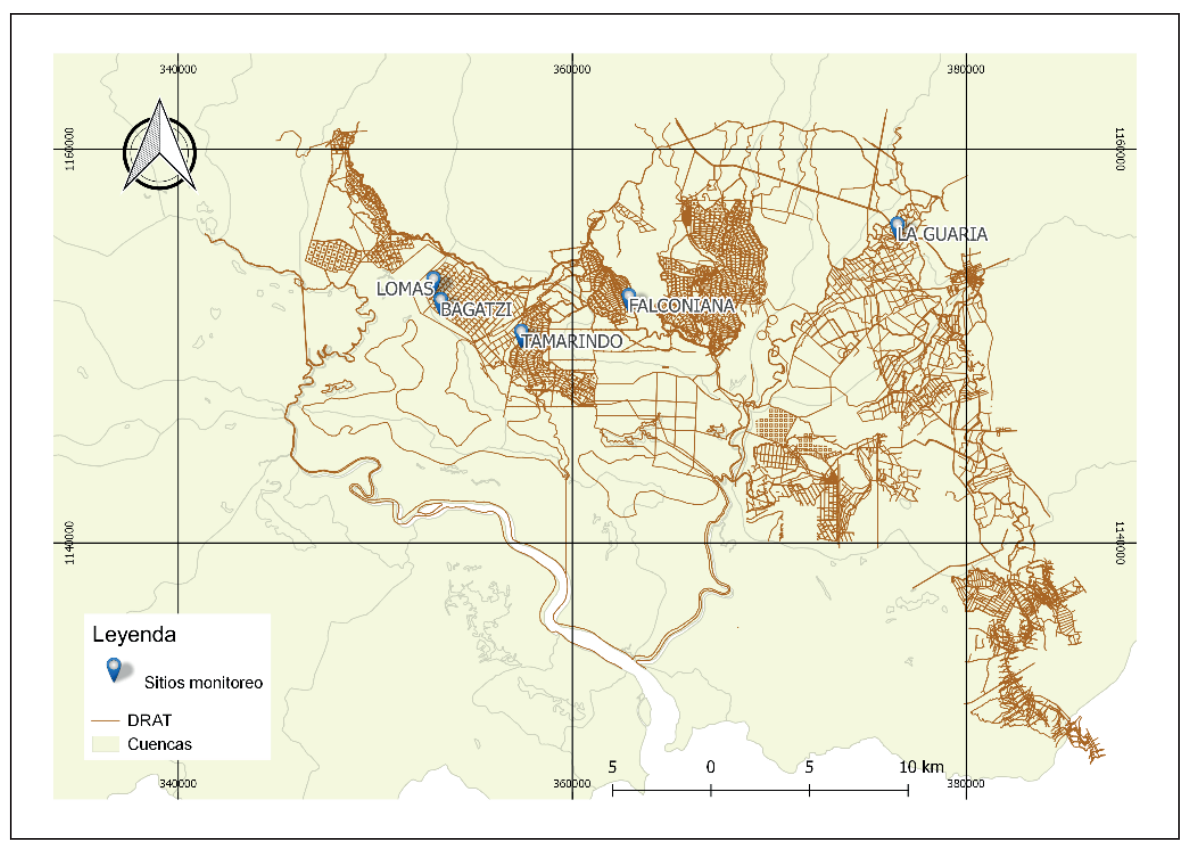

Figura 1. Sitios de monitoreo en el Distrito de Riego Arenal Tempisque, Guanacaste Fuente: elaboración propia, 2019.

Mediciones ambientales. La química y el flujo de agua fueron medidos en un punto único en el centro de los canales. Se tomó una muestra de agua de $2500 \mathrm{~mL}$ de en una botella de polipropileno, se transportó en hielo y se mantuvo en refrigeración para el análisis de DBO, DQO, amonio y fosfatos. El porcentaje de saturación de oxígeno, el pH y conductividad fueron medidos en el sitio utilizando la multisonda YSI 556MS. La velocidad de flujo se midió en el centro del canal usando un medidor de flujo portátil Global Water FP21 1.

Las muestras fueron analizadas según los protocolos establecidos en el laboratorio para Aguas y Especies Dulceacuícolas (LARED). Para el análisis de la demanda química de oxígeno la muestra de agua se oxidó con una solución sulfúrica caliente de dicromato potásico y sulfato de plata como catalizador. Asimismo, se determinó la concentración de iones de cobre (III) a una longitud de onda de $600 \mathrm{~nm}$ con la utilización de un espectrofotómetro Aquamate. Para la demanda química de oxígeno la materia orgánica en la muestra fue oxidada por una mezcla de ácidos crómico y sulfúrico en ebullición. Las muestras de agua residual se llevaron a reflujo en una solución ácida con un exceso conocido de $\mathrm{K} 2 \mathrm{Cr} 2 \mathrm{O} 7$. Una vez llevada a cabo la digestión se leyó en un espectofotómetro a $600 \mathrm{~nm}$. El amonio fue analizado mediante el método espectrofotométrico, el amonio en medio alcalino presente en la alícuota de la muestra reaccionó con el saliciliato e hipoclorito de sodio para dar un compuesto indofenólico verde esmeralda, cuya intensidad de color fue medida a $655 \mathrm{~nm}$. Para los fosfatos se siguió el método por ácido ascórbico Hach 8048 espectrofotométrico, el cual en un medio ácido el anión fosfato y el molibdeno amónico en presencia de tartrato de potasio y antimonio generan ácido fosfomolíbdico, el cual es reducido mediante ácido ascórbico y genera una coloración azul a una longitud de onda de $890 \mathrm{~nm}$.

Colección de macroinvertebrados. Los macroinvertebrados fueron recolectados utilizando el método de sustratos artificiales (Castillo 2010), los cuales fueron colonizados por los organismos durante cada mes.

Esta obra esta bajo una Licencia de Creative Commons Atribución NoComercial-SinDerivar Internacional 
Oriolus Revista Científica, ISSN: 2215-6100.1(1). Julio, 2020, pp 1-13

Arias Araya

Cada sustrato consistió en un bloque de arcilla con dimensiones $23 \times 11 \times 5 \mathrm{~cm}$ lineales dentro de una malla de nylon de $0,3 \mathrm{~cm}$. En cada sitio de muestreo se colocaron dos sustratos, se realizaron 7 muestreos en el Distrito de Riego Arenal Tempisque donde se muestrearon 70 sustratos. Los sitios de Paso Hondo y Lomas no fueron muestreados completamente debido al crecimiento de vegetación en el interior del canal.

Cada mes los sustratos y las mallas en campo fueron recolectados, lavados y trasladados al laboratorio, donde se almacenaron en alcohol 70 \% para ser analizadas. Los organismos fueron identificados por medio de claves taxonómicas disponibles y preservados en alcohol al $70 \%$.

Análisis de datos. Para los parámetros fisicoquímicos se estimaron los valores promedios, el máximo, el mínimo y la desviación estándar, en cada punto de muestreo, fueron organizados en tablas. Se utilizó la prueba de Shapiro Wilks para conocer la distribución de las variables y luego la prueba de Kruskal Wallis.

Para conocer la calidad del agua se estimó el Índice Holandés.

Para los macroinvertebrados se determinó la riqueza (número total de taxa, taxa familias), Tolerancia/Intolerancia (Índice BMWP) y la abundancia total.

\section{Resultados y discusión}

En la tabla 1 se aprecia los valores mínimos y máximos, así como el promedio y la desviación estándar de cada una de las variables medidas en cada canal. En el caso de la temperatura se observa que los valores obtenidos se encuentran dentro del rango permitido para vertido en cuerpos receptores (33601-MINAE-S) (15-40 ${ }^{\circ} \mathrm{C}$ ) en cada uno de los canales. El pH varía para el canal de Falconiana entre 7,21-9,10 siendo este último básico que podía comprometer la vida acuática, el pH ejerce una fuerte influencia sobre la toxicidad de ciertos parámetros químicos, tales como el amonio no ionizado, que se torna más abundante en $\mathrm{pH}$ alcalino, en los demás sitios se encuentran dentro de rango óptimo para la vida acuática $(6,5-8,5)$. En el caso del oxígeno disuelto los canales de Tamarindo y Bagatzí presentaron valores por debajo de $3 \mathrm{mg} / \mathrm{L}$ y $34 \%$ PSO, los otros sitios presentan valores más altos. La demanda bioquímica de oxígeno en cada uno de los canales muestreados se encuentra por debajo del límite máximo permisible en la legislación nacional (50 mg/L) mientras que la demanda química de oxígeno en los canales de Tamarindo, Bagatzí y La Guaria alcanzaron valores por encima del máximo permitido (150mg/L), la relación DQO/DBO para los tres canales descritos, anteriormente, expresan que el Índice de Biodegradabilidad es alto, lo que indica que la materia orgánica sea poco degradable. Según Orozco et al. (2011), entre la materia orgánica no biodegradable se encuentran los plaguicidas organoclorados, policlorobifenilos, hidrocarburos aromáticos policíclicos, celulosa, polvo de carbón, taninos y madera; estos materiales pueden permanecer largo período en las aguas y en algunos casos bioacumularse en los seres vivos por eso son conocidos como Compuestos Orgánicos Persistentes. Los nutrientes de amonio y fosfato presentaron valores por debajo del 0,5 mg/L. 
Oriolus Revista Científica, ISSN: 2215-6100.1(1). Julio, 2020, pp 1-13 Arias Araya

Tabla 1. Resumen de parámetros físicos y químicos en canales de drenaje del Distrito de Riego Arenal Tempisque. Fuente: elaboración propia

\begin{tabular}{|c|c|c|c|c|c|c|}
\hline Lugar & Variable & Unidad & Media & SD & Min & Máx. \\
\hline \multirow[t]{10}{*}{ Falconiana } & Temperatura & ${ }^{\circ} \mathrm{C}$ & 26,22 & 2,02 & 22,70 & 28,80 \\
\hline & $\mathrm{pH}$ & & 8,19 & 0,68 & 7,21 & 9,10 \\
\hline & Conductividad & $\mu \mathrm{S} / \mathrm{cm}$ & 117,33 & 22,75 & 91,90 & 150,30 \\
\hline & OD & $\mathrm{mg} / \mathrm{L} \bigcirc 2$ & 6,39 & 0,73 & 5,42 & 7,23 \\
\hline & PSO & $\%$ & 79,93 & 8,05 & 71,20 & 91,40 \\
\hline & Caudal & $\mathrm{m} 3 / \mathrm{s}$ & 0,07 & 0,066 & 0,03 & 0,19 \\
\hline & DBO & $\mathrm{mg} / \mathrm{L}$ & 13,36 & 6,67 & 2,28 & 26,56 \\
\hline & DQO & $\mathrm{mg} / \mathrm{L}$ & 44,00 & 31,28 & 19,00 & 110,00 \\
\hline & Amonio & mg/L N-NH4+ & 0,22 & 0,09 & 0,13 & 0,39 \\
\hline & Fósforo & mg/L P-PO43- & 0,13 & 0,06 & 0,05 & 0,21 \\
\hline \multirow[t]{10}{*}{ Tamarindo } & Temperatura & ${ }^{\circ} \mathrm{C}$ & 26,51 & 2,30 & 22,50 & 28,90 \\
\hline & $\mathrm{pH}$ & & 7,95 & 0,60 & 7,28 & 8,81 \\
\hline & Conductividad & $\mu \mathrm{S} / \mathrm{cm}$ & 195,88 & 78,87 & 116,60 & 323 \\
\hline & OD & $\mathrm{mg} / \mathrm{L} \bigcirc 2$ & 5,06 & 1,69 & 2,70 & 7,13 \\
\hline & PSO & $\%$ & 66,03 & 22,99 & 34,00 & 97,20 \\
\hline & Caudal & $\mathrm{m} 3 / \mathrm{s}$ & 0,10 & 0,08 & 0,04 & 0,28 \\
\hline & DBO & $\mathrm{mg} / \mathrm{L}$ & 14,86 & 10,46 & 3,04 & 33,63 \\
\hline & DQO & $\mathrm{mg} / \mathrm{L}$ & 54,00 & 51,20 & 14,40 & 161,00 \\
\hline & Amonio & mg/L N-NH4+ & 0,26 & 0,15 & 0,14 & 0,60 \\
\hline & Fósforo & mg/L P-PO43- & 0,20 & 0,09 & 0,09 & 0,37 \\
\hline \multirow[t]{10}{*}{ Bagatzí } & Temperatura & ${ }^{\circ} \mathrm{C}$ & 26,59 & 2,18 & 22,80 & 28,70 \\
\hline & $\mathrm{pH}$ & & 7,58 & 0,32 & 7,23 & 8,01 \\
\hline & Conductividad & $\mu \mathrm{S} / \mathrm{cm}$ & 266,93 & 66,71 & 155,80 & 360,00 \\
\hline & OD & $\mathrm{mg} / \mathrm{L} \mathrm{O2}$ & 4,61 & 1,17 & 2,40 & 6,20 \\
\hline & PSO & $\%$ & 59,35 & 15,97 & 28,00 & 79,00 \\
\hline & Caudal & $\mathrm{m} 3 / \mathrm{s}$ & 0,50 & 0,21 & 0,30 & 0,75 \\
\hline & DBO & $\mathrm{mg} / \mathrm{L}$ & 12,23 & 6,84 & 1,52 & 21,98 \\
\hline & DQO & $\mathrm{mg} / \mathrm{L}$ & 67,27 & 66,75 & 10,54 & 208,10 \\
\hline & Amonio & mg/L N-NH4+ & 0,27 & 0,13 & 0,10 & 0,42 \\
\hline & Fósforo & mg/L P-PO43- & 0,18 & 0,09 & 0,06 & 0,30 \\
\hline
\end{tabular}


Oriolus Revista Científica, ISSN: 2215-6100.1(1). Julio, 2020, pp 1-13 Arias Araya

\begin{tabular}{|c|c|c|c|c|c|c|}
\hline \multirow[t]{10}{*}{ La Guaria } & Temperatura & ${ }^{\circ} \mathrm{C}$ & 27,29 & 1,18 & 25,00 & 28,50 \\
\hline & $\mathrm{pH}$ & & 7,76 & 0,42 & 7,25 & 8,43 \\
\hline & Conductividad & $\mu S / c m$ & 129,53 & 30,54 & 86,00 & 175,00 \\
\hline & OD & $\mathrm{mg} / \mathrm{L} \mathrm{O2}$ & 5,61 & 0,95 & 4,30 & 6,95 \\
\hline & PSO & $\%$ & 68,12 & 11,51 & 51,00 & 88,20 \\
\hline & Caudal & $\mathrm{m} 3 / \mathrm{s}$ & 0,05 & 0,02 & 0,03 & 0,08 \\
\hline & DBO & $\mathrm{mg} / \mathrm{L}$ & 12,77 & 9,99 & 2,28 & 33,80 \\
\hline & DQO & $\mathrm{mg} / \mathrm{L}$ & 56,99 & 62,45 & 11,00 & 190,00 \\
\hline & Amonio & mg/L N-NH4+ & 0,19 & 0,10 & 0,06 & 0,36 \\
\hline & Fósforo & mg/L P-PO43- & 0,07 & 0,03 & 0,04 & 0,13 \\
\hline \multirow[t]{10}{*}{ Lomas } & Temperatura & ${ }^{\circ} \mathrm{C}$ & 32,12 & & & \\
\hline & $\mathrm{pH}$ & & 8,21 & & & \\
\hline & Conductividad & $\mu S / c m$ & 495,00 & & & \\
\hline & OD & $\mathrm{mg} / \mathrm{L} \mathrm{O2}$ & 4,15 & & & \\
\hline & PSO & $\%$ & 50,13 & & & \\
\hline & Caudal & $\mathrm{m}^{3} / \mathrm{s}$ & 0,04 & & & \\
\hline & DBO & $\mathrm{mg} / \mathrm{L}$ & 28,35 & 0,44 & 28,00 & 28,84 \\
\hline & DQO & $\mathrm{mg} / \mathrm{L}$ & 104,89 & 1,60 & 103,61 & 106,69 \\
\hline & Amonio & $\mathrm{mg} / \mathrm{L} \mathrm{N}-\mathrm{NH}_{4}^{+}$ & 0,20 & & & \\
\hline & Fósforo & $\mathrm{mg} / \mathrm{L} \mathrm{P}-\mathrm{PO}_{4}^{3-}$ & 0,05 & 0,01 & & \\
\hline \multirow{10}{*}{$\begin{array}{c}\text { Paso Hon- } \\
\text { do }\end{array}$} & Temperatura & ${ }^{\circ} \mathrm{C}$ & 25,00 & & & \\
\hline & $\mathrm{pH}$ & & 7,18 & & & \\
\hline & Conductividad & $\mu S / c m$ & 91,40 & & & \\
\hline & $O D$ & $\mathrm{mg} / \mathrm{L} \mathrm{O}_{2}$ & 4,40 & & & \\
\hline & PSO & $\%$ & 55,60 & & & \\
\hline & Caudal & $\mathrm{m}^{3} / \mathrm{s}$ & 0,01 & & & \\
\hline & DBO & $\mathrm{mg} / \mathrm{L}$ & 18,89 & 0,61 & 18,24 & 19,45 \\
\hline & DQO & $\mathrm{mg} / \mathrm{L}$ & 32,91 & 2,29 & 31,00 & 35,45 \\
\hline & Amonio & $\mathrm{mg} / \mathrm{L} \mathrm{N}-\mathrm{NH}_{4}^{+}$ & 0,15 & & & \\
\hline & Fósforo & $\mathrm{mg} / \mathrm{L} \mathrm{P}-\mathrm{PO}_{4}{ }^{3-}$ & 0,16 & & & \\
\hline
\end{tabular}

Nota: pH: Potencial de hidrógeno, OD: Oxígeno disuelto, PSO: Porcentaje de saturación de oxígeno, DBO: Demanda bioquímica de oxígeno (5,20), DQO: Demanda química de oxígeno.Fuente: elaboración propia, 2019. 
Oriolus Revista Científica, ISSN: 2215-6100.1 (1). Julio, 2020, pp 1-13

Arias Araya

Según la prueba de Shapiro Wilks los datos no tienen una distribución normal. Aplicando la prueba de Kruskal Wallis se obtiene que la temperatura $(p=0,023)$, el $\mathrm{pH}(\mathrm{p}=0,0022)$, oxígeno disuelto $(p=0,0001)$, PSO $(p=0,0004)$, conductividad $(p<0,0001)$, caudal $(p<0,0001)$ y fósforo $(p<0,0001)$ presentan diferencias estadísticamente significativas entre las medianas de las variables determinadas en cada uno de los canales muestreados. Esto se relaciona con los cultivos y el manejo de cada terreno en los diferentes sitios.

Para conocer la calidad del agua en cuanto a materia orgánica se aplicó el Índice Holandés, que contempla los parámetros de DBO, PSO y amonio, a pesar de que este índice es para cuerpos superficiales se utilizó con el fin de compararlo con los datos obtenidos. En la figura 2 se observa los valores promedios obtenidos en cada canal durante los meses de estudio. En el mes de marzo los cuatro sitios se categorizan en contaminación moderada, en el mes de abril únicamente el canal de Tamarindo pasa a contaminación severa, en el mes de junio Falconiana y Tamarindo bajan a contaminación incipiente, mientras Bagatzí y La Guaria se mantienen en la misma clase. Durante julio la contaminación es moderada para todos los canales y en agosto Falconiana, Tamarindo y la Guaria bajan a contaminación incipiente. Por último, en el mes de noviembre Falconiana sube de clase a contaminación moderada, mientras los demás se mantienen.

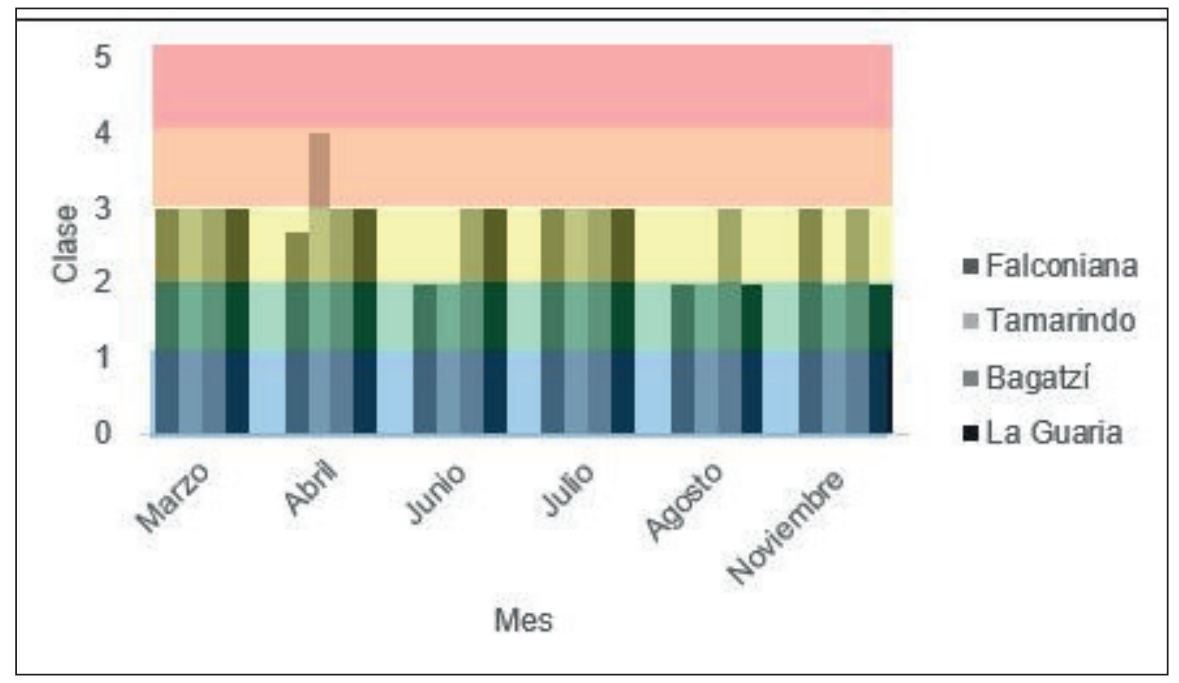

Figura 2. Calidad de las aguas en los canales de drenaje del DRAT según el Índice Holandés. Fuente: elaboración propia, 2019.

Para el análisis de la calidad de un cuerpo de agua pueden utilizarse diferentes métodos, tanto fisicoquímicos como biológicos. El mayor problema de los parámetros fisicoquímicos radica en que sus resultados son siempre puntuales. Por lo que no tienen en cuenta otras alteraciones del ecosistema acuático que pueden afectar a sus componentes.

Para complementar a los anteriores se recurre a métodos biológicos, basados en las comunidades de organismos acuáticos, ya que estos reaccionan ante alteraciones en la calidad de las aguas cambiando su composición específica. Aportan una visión, no solo puntual, sino también histórica (Gutiérrez-Fonseca y Lorion, 2014). 
Oriolus Revista Científica, ISSN: 2215-6100.1(1). Julio, 2020, pp 1-13

Arias Araya

En la figura 3A se observa la abundancia de taxa en cada uno de los canales, los canales con mayor abundancia de macroinvertebrados fueron Tamarindo (T2), Bagatzí (B3) y Falconiana (F1). Con respecto a la riqueza se observó que Falconiana (F1) y La Guaria (G4) presentan mayor diversidad, esto se relaciona con la concentración de oxígeno disuelto, ya que ambos canales no presentaron niveles por debajo de 5ppm.

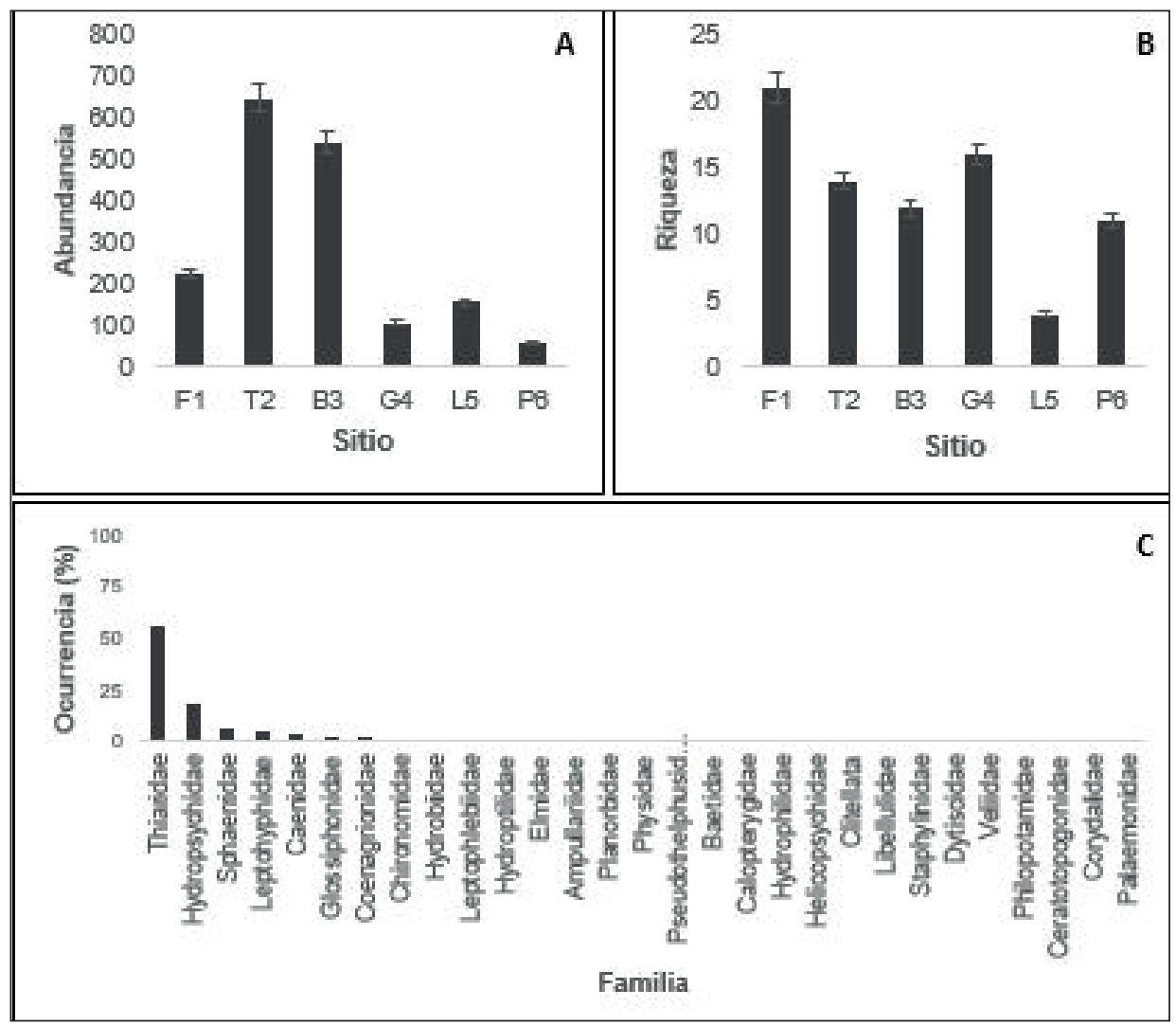

Figura 3. Macroinvertebrados bentónicos por sitio en el Distrito de Riego Arenal Tempisque. (A) Abundancia. (B) Riqueza. (C) Ocurrencia de las familias encontradas. Fuente: elaboración propia, 2019.

Durante el estudio se registraron en total 21 familias y un total de 1736 individuos. La familia con más ocurrencia fue Thiaridae (56\%), estas especies pueden encontrarse en agua dulce corriente o estancada, se encuentran en sedimentos blandos y su alimentación se basa en perífito. Además, pueden soportar sequías periódicas, al enterrarse más profundamente en el sedimento (Bouchet et al., 2005). Según Hahn-vonHessberg, Toro, Grajales-Quintero, Duque-Quintero, Serna-Uribe (2009) las especies de Thiaridae están relacionadas con los bajos niveles de oxígeno disuelto, parámetro que predomina en los canales estudiados. La familia Hydropsychidae se encontró con una ocurrencia del $18,3 \%$, es una de las familias dominantes en aguas corrientes, tanto por su número, como por su diversidad (Angrisano y Korob, 2001), dada su capacidad para colonizar diferentes tipos de sustratos (roca, arena, grava, hojarasca), tanto en rápidos como en remansos, y a su capacidad para tolerar diferentes tipos de ambientes, desde aguas completamente limpias hasta aguas con algún grado de intervención antrópica (López, 2007). 
Oriolus Revista Científica, ISSN: 2215-6100.1(1). Julio, 2020, pp 1-13

Arias Araya

Según la prueba de Kruskal Wallis no existe diferencia significativa entre las familias y los canales, por lo que puede inferirse que el comportamiento de los canales es el mismo, ya que se siembran los mismos cultivos. Los patrones de distribución indican que factores como el caudal ejercen una marcada influencia en la comunidad béntica; condiciones estables del caudal favorecen la presencia de mayor número de microhábitats (Mosquera, Bejatano, Asprilla, 2006) y sus variaciones determinan

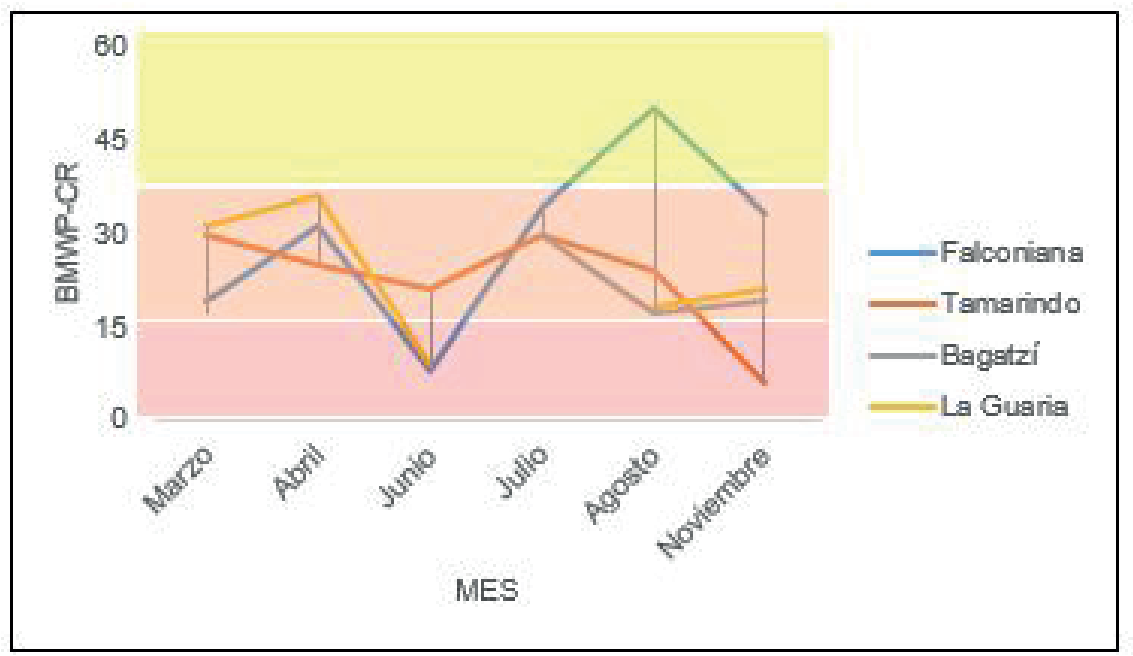

Figura 4. Calidad de las aguas en los canales de drenaje del DRAT según el índice BMWP-CR. Fuente: elaboración propia, 2019.

una regresión en la sucesión de las comunidades de macroinvertebrados.

En la figura 4 se muestra la calidad de las aguas en los canales de drenaje del DRAT según el Índice BMWP-CR, en donde valores por debajo de 15 representan aguas de calidad muy mala, extremadamente contaminadas; entre 16 y 35 aguas de calidad mala, muy contaminadas; y entre 36-60 aguas de calidad mala, contaminadas.

\section{Conclusiones}

La descarga de agroquímicos y las prácticas de conservación de suelos son las principales actividades que afectan la estructura de la comunidad de macroinvertebrados y la calidad del agua, los distritos de Falconiana y Tamarindo son los que poseen más actividad agrícola durante el año.

Se identificaron 10 órdenes de macroinvertebrados: Mollusca, Odonata, Coleoptera, Annelida, Hemiptera, Trichoptera, Diptera, Ephemeroptera, Megaloptera y Crustáceo en los canales de drenaje.

La presencia de las familias Planorbidae, Planaridae, Libellulidae y Hydroptilidae se relaciona con alto porcentaje de saturación de oxígeno. Por el contrario, las familias Thiaridae y Chironomidae están relacionadas con bajos niveles de oxígeno.

Los métodos biológicos y fisicoquímicos son complementarios, el uso de estos métodos permite ahorro de tiempo y mejor utilización de los recursos económicos disponibles para la gestión de los recursos hídricos. La legislación ambiental no ha tenido en cuenta la gran utilidad e importancia de los 
Oriolus Revista Científica, ISSN: 2215-6100.1 (1). Julio, 2020, pp 1-13

Arias Araya

métodos biológicos, en especial los relacionados con el empleo de los macroinvertebrados acuáticos como bioindicadores.

\section{Bibliografía}

Angrisano, E. B., Korob, P.G. "Trichoptera". En: Guía para la determinación de los artrópodos bentónicos sudamericanos. Serie: investigaciones de la UNT editado por Fernández, H. R. \& E. Domínguez, 55-92. Tucumán, Argentina: Ciencias Exactas y Naturales-Universidad Nacional de Tucumán, 2001.

Armitage, P.D., Szoszkiewicz, K., Blackburn, J.H., Nesbitt, I. "Ditch communities: a major contributor to floodplain biodiversity". Aquatic Conservation: Marine and Freshwater Ecosystems 13 (2003): 165-185.

Bonada, N., Prat, N., Resh, V. H., \& Statzner, B. "Developments in aquatic insect biomonitoring: A comparative analysis of recent approaches". Annual Review of Entomology 51, (2006): 495-523.

Bouchet, Philippe., Rocroi, Jean-Pierre., Frýda, Jiri., Hausdorf, Bernard., Ponder, Winston., Valdés, Ángel., Warén, Anders. "Classification and nomenclator of gastropod families". Malacologia. Hackenheim, Germany: ConchBooks 47, N.1-2 (2005): 1-397. ISBN 3-925919-72-4. ISSN 0076-2997.

Castillo, L. E. Pesticide impact of intensive banana production on aquatic ecosystems in Costa Rica. Stockholm, Sweden: Stockholm University, 2010.

Clarke, Stewart. J. "Conserving freshwater biodiversity: The value, status andmanagement of high quality ditch systems". Journal for Nature Conservation, 24 (2014): 93-100. http://dx.doi.org/10.1016/j.jnc.2014.10.003

Gutiérrez-Fonseca, P., Lorion, C. "Application of the BMWP-Costa Rica biotic index in aquatic biomonitoring: sensitivity to collection method and sampling intensity". Rev Biol Trop., 62 N. 2 (2014): 275-289.

Hahn-vonHessberg, C., Toro, R., Grajales-Quintero, A., Duque-Quintero, G., Serna-Uribe, L. (2009). "Determinación de la calidad del agua mediante indicadores biológicos y fisicoquímicos, en la estación piscícola, Universidad de Cladas, Colombia". bol.cient.mus.hist.nat, 13, N. 2 (2009): 89-105.

Hanson, P., Springer, M., \& Ramirez, A. "Introducción a los grupos de macroinvertebrados acuáticos". Rev. Biol. Trop, (2010): 3-37.

Herzon, I., Helenius, J. "Agricultural drainage ditches, their biological importance and functioning". Biological Conservation, 141 (2008): 1171-1183

López, E. Análisis faunístico de las larvas del orden Trichoptera en la cuenca del río Prado y la subcuenca de Amoyá (Tolima-Colombia). Trabajo de grado (Biólogo). Universidad del Tolima, Facultad de Ciencias Básicas, Departamento de Biología, lbagué, 2007.

Mosquera, Z., Bejatano, D., Asprilla, S. Estudio del orden Trichoptera (Insecta) en dos ecosistemas lóticos del municipio de Quibdo, Chocó-Colombia. En: Memorias VII Seminario Colombiano de Limnología y I Reunión Internacional sobre Ríos y Humedales Neotropicales editado por F. Villa, C. Rivera, G. Flórez, M. Núñez \& X. Carranza, 85-91. León Gráficas, Ibagué, 2006.

Esta obra esta bajo una Licencia de Creative Commons Atribución NoComercial-SinDerivar Internacional 


\section{Oriolus Revista Científica, ISSN: 2215-6100.1(1). Julio, 2020, pp 1-13}

Arias Araya

Orozco, C., Pérez, A., González, M.N., Rodríguez, F., Alfayate, J. Contaminación ambiental. Una visión desde la química. Ediciones Paraninfo, 2011. ISBN: 978-84-9732-178-5. 78

Painter, D. "Macroinvertebrate distributions and the conservation value of aquatic Coleoptera, Mollusca and Odonata in the ditches of traditionally managed and grazing fen at Wicken Fen, UK". Journal of Applied Ecology, 36 (1999): 33-48.

Presidente de la República, Ministerio de Ambiente y Ministra de Salud. Decreto Ejecutivo: 33903 Reglamento para la Evaluación y Clasificación de la Calidad de Cuerpos de Agua Superficiales. Gaceta 178. San José, Costa Rica, 2007.

SENARA. Demandas de agua en el Distrito de Riego Arenal Tempisque, 2018.

Vega-Prado Leiva, J. Evaluación de la calidad de agua del Río Yeguare mediante el uso de macro invertebrados como indicadores biológicos. Honduras: Desarrollo Socioeconómico y Ambiente, 2004.

Verdonschot, R., Keizer-Vlek, H., Verdonschot, P. (2012). "Development of a multimetric index based on macroinvertebrates for drainage ditch networks in agricultural areas". Ecological Indicators. 13 (2012): 232-242.

Vörösmarty, C. J., Mclntyre, P. B., Gessner, M. O., Dudgeon, D., Prusevich, A., Green, P., Davies, P. M. (2010). "Global threats to human water security and river biodiversity". Nature 467, (2010): 555-561. 